\title{
Bioanalysis
}

\section{Bioanalytical methods for the quantification of cis-permethrin and trans- permethrin in biological samples}

\author{
"Reliable, sensitive and accurate analytical methods are necessary to \\ measure concentrations of PERM in biological samples for human risk \\ assessment and research purposes."
}

First draft submitted: 20 July 2017; Accepted for publication: 2 August 2017;

Published online: 21 October 2017

Keywords: gas chromatography $\bullet$ negative chemical ionization $\bullet$ permethrin

Permethrin (PERM) is a synthetic type I pyrethroidal pesticide with broad-spectrum insecticide activity. This compound has been widely used over the past 50 years in agriculture, indoor pest control products, lotions or shampoos for head lice treatment, mosquito control and has been incorporated into the fabric of soldiers' uniforms to combat insects [1].

PERM has relatively low volatility and can be readily adsorbed onto surfaces such as furniture, walls and carpets. Therefore, there are significant concerns related to exposure to low levels of PERM, which can occur routinely. Following domestic or agricultural uses of PERM, a significant amount of PERM residue may enter into the ecosystem which poses a potential threat to both human health and to other nontargeted species [2].

Human exposure to PERM can occur through inhalation, oral or dermal routes and due to its lipophilic nature, can accumulate in the brain and persist long after exposure $[3,4]$. Although PERM has low mammalian toxicity, it has been shown to cause a variety of adverse events in animals and humans such as neurotoxicity, immunotoxicity, cardiotoxicity, genotoxicity, fetotoxicity and hepatotoxicity [5]. Therefore, the availability of sensitive and accurate bioanalytical methods for the quantitation of this commonly used pesticide is essential to evaluate its toxic effects on mammals. Since deficits in cognition due to prolonged subthreshold exposures are particularly of interest (as these have been observed in many pesticides that target the cholinesterase pathway), the ability to accurately measure PERM in the brain is important. It is also ideal to be able to correlate these brain exposures to plasma or urine concentrations, since these are easily accessible fluids following exposures in humans.

Commercially available PERM exists as a mixture of the cis-isomers and trans-isomers. Different methods of production lead to different ratios of the cis and trans-isomers, such as $40: 60,80: 20$ or $25: 75$. The two isomers have different rates of metabolism, activity and stability. Therefore, it is important to be able to assess the individual contributions of each isomer when measuring PERM. Currently, only two methods have been reported for the simultaneous measurement of these two isomers in rat plasma and the brain $[6,7]$. The chromatographic and sample preparation details of these methods will be discussed in the following section.

\section{Bioanalytical methods}

PERM is a nonpolar, semi volatile compound with electronegative atoms in its structure that make it a perfect target for gas chromatography mass spectrometry (GC-MS) analysis in negative chemical ionization (NCI) mode. Several analytical strategies for the separation and determination of PERM in biological matrices have been reported [6-12]. However, using GC-NCI-MS has provided the most sensitive method for the bioanalysis of PERM

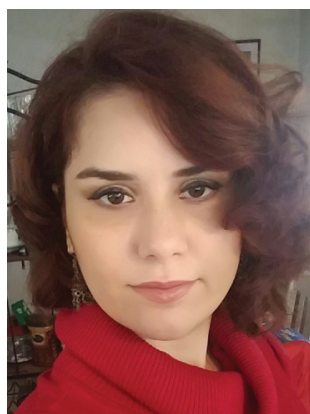

Shirin Hooshfar Department of Pharmaceutical \& Biomedical Sciences, College of Pharmacy, The University of Georgia, Athens, GA 30602-2352, USA

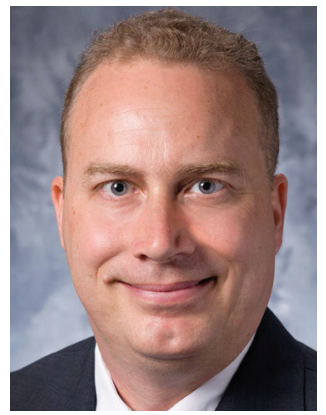

Michael G Bartlett

Author for correspondence: Department of Pharmaceutical \& Biomedical Sciences, College of Pharmacy, The University of Georgia, Athens, GA 30602-2352, USA

Tel.: + 17065425390

Fax: + 17065425358

mgbart@uga.edu

newlands press part of 
or its two isomers [7,9]. Additionally, the high number of theoretical plates available when using capillary stationary phases in GC allowed for an easy separation of the cis-isomers and trans-isomers in relatively short method run times [7]. Ramesh and Ravi used both NCI and electron ionization for the quantitation of PERM in whole blood $[9,13]$. Using NCI they were able to decrease the LOQ of method to $0.02 \mathrm{ng} / \mathrm{ml}$ from the $0.2 \mathrm{ng} / \mathrm{ml}$ levels that were achieved when using electron ionization.

Different techniques have been developed for the extraction and cleanup of PERM from biological samples. Hydrophilic lipophilic balance columns were used by Leng and Gries for the extraction of PERM from plasma [10]. In this method, diluted plasma was loaded on a cartridge and after several washing steps, PERM was eluted by using a $1: 1(\mathrm{v} / \mathrm{v})$ mixture of hexane:dichloromethane. The sample was finally analyzed by high-resolution GC-NCI-MS with a method LOD of $5 \mathrm{ng} / \mathrm{ml}$. This method was modified by OgataKawata et al. for quantitation of cis-PERM and transPERM in whole blood [14]. By increasing the dilution factor by five-times and changing the elution solvent to toluene, they managed to quantify the PERM isomers with a method LOQ of $0.6 \mathrm{ng} / \mathrm{ml}$. However, this method has a very long run time $(30 \mathrm{~min})$ that is less suited for the routine analysis of a large number of samples.

\section{"To date, GC-MS-NCl is the most sensitive approach for the determination of PERM isomers."}

Lestremau et al. developed a GC-MS method for the simultaneous determination of PERM isomers and their associated metabolites in different biological rat matrices and fluids (whole blood, red blood cells, plasma, brain, liver, muscle, testes, kidneys, fat and feces) [6]. They used a protein precipitation technique (with $1 \mathrm{ml}$ methanol and $1 \mathrm{ml}$ of concentrated hydrochloric acid $[10 \mathrm{M}]$ ) to extract these compounds from biological samples and then used ion trap tandem MS to quantify them. This method was also used by Willemin et al. for physiologically-based pharmacokinetic (PBPK) modeling of the cis-PERM and trans-PERM in rats [15]. However, the use of strong inorganic acids such as the hydrochloric acid in this method poses a substantial risk of stationary phase damage, especially during the analysis of large batches of samples. Also, the methods developed by Ogata-Kawata and Hooshfar et al. are 80- and 250-fold more sensitive for both isomers, respectively [7,14].

Biological matrices contain lipids, proteins and other non-specific constituents that are co-extracted with the analyte and may adsorb in GC inlet ports and columns [16,17]. Hooshfar et al. compared the ability of different sample preparation approaches such as protein precipitation, liquid-liquid extraction and solid-phase extraction for decreasing these interfering compounds in brain and plasma samples [7]. Ultimately they introduced a two-step liquid-liquid extraction method by using $1.2 \mathrm{ml}$ diisopropyl ether and $100 \mu \mathrm{l}$ acetonitrile. This method is the most sensitive analytical method that has been reported for the simultaneous determination of cis-PERM and trans-PERM in rat plasma and brain (LOQ: $0.2 \mathrm{ng} / \mathrm{ml}$ ). Also, for the first time only $100 \mu \mathrm{l}$ of the biological sample for analysis of PERM isomers was used. The use of low-volume biological samples is essential for supporting toxicokinetic experiments in small rodents, where as many as ten serial samples of blood are drawn at time points over a $12-96 \mathrm{~h}$ period $[16,17]$.

The PERM isomers are unstable in biological samples $[7,18]$. This is the most challenging problem in analysis of these two isomers and should be seriously considered during method development. Most of the studies in the literature use formic acid or sodium fluoride to improve sample stability by inhibiting esterases [6-7,18]. Hooshfar et al. found that neither of these alone or in combination with each other can prevent sample degradation during bench top or freezethaw stability tests [7]. Furthermore, formic acid can adversely affect the sensitivity of the method. They proposed the use of the immediate addition of sodium fluoride after sampling to manage this problem.

To date, GC-MS-NCI is the most sensitive approach for the determination of PERM isomers. However, there is no commercially available stable isotope labeled versions of PERM that can be used as an internal standard in NCI mode [7]. This is because current pyrethroids are labeled in the phenoxybenzyl moiety. When conducting NCI with methane, PERM undergoes fragmentation following electron capture to generate a dichloroethenyl cyclopropane oxonium ion. Therefore, the isotopically labeled portion of the molecule is lost. Although the structure of other pyrethroids pesticides are similar enough to use them as an internal standard, they have different biological stabilities. For example, Hooshfar et al. used transfluthrin as the internal standard and added it immediately after sampling to help reduce method variability. Nevertheless, transfluthrin is only slightly more stable than PERM in both plasma and brain [7,19]. Therefore, having an isotope-labeled internal standard with exactly similar physicochemical properties to the analyte would be beneficial for increasing the accuracy of future bioanalytical methods and synthesis of these compounds should be considered. It is also possible that LC-MS methods could be developed for the analysis of PERM. Although to date the separation of the two isomers has not been demonstrated and it is not clear if LC-MS can achieve the low levels of detection that has currently been achieved using GC-NCI-MS. 


\section{Conclusion}

Reliable, sensitive and accurate analytical methods are necessary to measure concentrations of PERM in biological samples for human risk assessment and research purposes. Due to the relatively short plasma half-life of PERM, most of the developed bioanalytical methods focused on measuring its metabolites in urine and the quantity of published reports for the determination of PERM in plasma, blood or tissues are quite limited. Also the search of literature cited here indicates few reports about the simultaneous measurement of cisPERM and trans-PERM in biological samples. Different types of analytical approaches have been used for the determination of PERM and the highest method sensitivity has been achieved by GC-NCI-MS technique.

\section{References}

1 Gammon DW. Permethrin A2 - Wexler Philip. In: Encyclopedia of Toxicology (3rd Edition), Academic Press, Oxford, UK, 808-811 (2014).

2 Stout Ii DM, Bradham KD, Egeghy PP et al. American Healthy Homes Survey: a national study of residential pesticides measured from floor wipes. Envir. Sci. Tech. 43(12), 4294-4300 (2009).

3 Carloni M, Nasuti C, Fedeli D et al. The impact of early life permethrin exposure on development of neurodegeneration in adulthood. Exp. Gerontol. 47(1), 60-66 (2012).

4 Nasuti C, Brunori G, Eusepi P, Marinelli L, Ciccocioppo R, Gabbianelli R. Early life exposure to permethrin: a progressive animal model of Parkinson's disease. J. Pharmacol. Toxicol. Methods 83, 80-86 (2017).

5 Wang X, Martinez MA, Dai M et al. Permethrin-induced oxidative stress and toxicity and metabolism. A review. Environ. Res. 149, 86-104 (2016).

6 Lestremau F, Willemin ME, Chatellier C, Desmots $S$, Brochot C. Determination of cis-permethrin, transpermethrin and associated metabolites in rat blood and organs by gas chromatography-ion trap mass spectrometry. Anal. Bioanal. Chem. 406(14), 3477-3487 (2014).

7 Hooshfar S, Gullick DR, Linzey MR et al. Simultaneous determination of cis-permethrin and trans-permethrin in rat plasma and brain tissue using gas chromatography-negative chemical ionization mass spectrometry. J. Chromatogr. B Analyt. Technol. Biomed. Life Sci. 1060, 291-299 (2017).

8 Oehler D. Gas-liquid chromatographic determination of permethrin in bovine tissues. J. Assoc. Offic. Analyt. Chemists 62(6), 1309-1311 (1979).

9 Ramesh A, Ravi PE. Negative ion chemical ionization-gas chromatographic-mass spectrometric determination of residues of different pyrethroid insecticides in whole blood and serum. J. Anal. Toxicol. 28(8), 660-666 (2004).

10 Leng G, Gries W. Determination of pyrethroids in blood plasma and pyrethroid/pyrethrin metabolites in urine by gas
The PERM isomers are unstable in biological samples and this is the major concern in the bioanalysis of these compounds. Using an isotope labeled internal standard can be a promising approach to overcome this problem by reducing the method variability.

\section{Financial \& competing interests disclosure}

The authors have no relevant affiliations or financial involvement with any organization or entity with a financial interest in or financial conflict with the subject matter or materials discussed in the manuscript. This includes employment, consultancies, honoraria, stock ownership or options, expert testimony, grants or patents received or pending, or royalties.

No writing assistance was utilized in the production of this manuscript.

chromatography-mass spectrometry and high-resolution GC-MS. Pesticide Protocols 19, 17-33 (2006).

11 Santos MA, Rodrigues MV, Áreas MA, Reyes FG. Deltamethrin and permethrin in the liver and heart of Wistar rats submitted to oral subchronic exposure. $J$. Brazilian Chemical Soc. 22(5), 891-896 (2011).

12 Abu-Qare AW, Abou-Donia MB. A solid phase extraction reversed-phase HPLC method for the simultaneous determination of methoprene, permethrin and selected metabolites in rat plasma and urine. Biomed. Chromatogr. 15(7), 464-470 (2001).

13 Ramesh A, Ravi PE. Electron ionization gas chromatography-mass spectrometric determination of residues of thirteen pyrethroid insecticides in whole blood. J. Chromatogr. B Analyt. Technol. Biomed. Life Sci. 802(2), 371-376 (2004).

14 Ogata-Kawata H, Matsuda M, Onda N et al. Direct analysis of permethrins in human blood by SPE-GC/MS. Chromatography 28(3), 119 (2007).

15 Willemin M-E, Desmots S, Le Grand R et al. PBPK modeling of the cis-and trans-permethrin isomers and their major urinary metabolites in rats. Toxicol. Appl. Pharm. 294, 65-77 (2016).

16 Gullick DR, Mott KB, Bartlett MG. Chromatographic methods for the bioanalysis of pyrethroid pesticides. Biomed. Chromatogr. 30(5), 772-789 (2016).

17 Gullick D, Popovici A, Young HC et al. Determination of deltamethrin in rat plasma and brain using gas chromatography-negative chemical ionization mass spectrometry. J. Chromatogr. B Analyt. Technol. Biomed. Life Sci. 960, 158-165 (2014).

18 Leng G, Kühn K-H, Idel H. Biological monitoring of pyrethroids in blood and pyrethroid metabolites in urine: applications and limitations. Sci. Total Environ. 199(1), 173-181 (1997).

19 Hooshfar S, Mortuza TB, Rogers CA et al. Negative chemical ionization gas chromatography-mass spectrometry of transfluthrin in rat plasma and brain. Rapid Commun. Mass Spectrom. 31(19), 112-117 (2017). 